\title{
Transversality of holomorphic mappings between real hypersurfaces in different dimensions
}

\author{
M.S. Baouendi, Peter Ebenfelt and Linda P. Rothschild
}

\begin{abstract}
In this paper, we consider holomorphic mappings between real hypersurfaces in different dimensional complex spaces. We give a number of conditions that imply that such mappings are transversal to the target hypersurface at most points.
\end{abstract}

\section{Introduction and main results}

In this paper, we consider holomorphic mappings between real hypersurfaces in different dimensional complex spaces. We shall always assume that the dimensions of the complex spaces are at least two. We give a number of conditions implying that such mappings are transversal to the target hypersurface at most points. Recall that if $U$ is an open subset of $\mathbb{C}^{n+1}$ (with $n \geq 1$ ), $H$ a holomorphic mapping $U \rightarrow \mathbb{C}^{n^{\prime}+1}$, and $M^{\prime}$ a real hypersurface through a point $H(p)$ for some $p \in U$, then $H$ is said to be transversal to $M^{\prime}$ at $H(p)$ if

$$
T_{H(p)} M^{\prime}+d H\left(T_{p} \mathbb{C}^{n+1}\right)=T_{H(p)} \mathbb{C}^{n^{\prime}+1},
$$

where $T_{p} \mathbb{C}^{n+1}$ and $T_{H(p)} M^{\prime}$ denote the real tangent spaces of $\mathbb{C}^{n+1}$ and $M^{\prime}$ at $p$ and $H(p)$, respectively. (We mention that the notion of transversality of a mapping to a hypersurface coincides with that of $\mathrm{CR}$ transversality; cf. [15].) We shall assume that there is a real hypersurface $M \subset U$ such that $H(M) \subset M^{\prime}$. Then transversality at a point $H(p)$, for $p \in M$, is equivalent to the nonvanishing at $p$ of the normal derivative of the real function $u:=$ $\rho^{\prime} \circ H$, where $\rho^{\prime}=0$ is a local defining equation for $M^{\prime}$ near $H(p)$. Hence a result on transversality can be regarded as a type of Hopf Lemma.

The equidimensional case (i.e., $n=n^{\prime}$ ) has been considered by many authors; we mention here the papers [2, 5, 7-10, 15, 17, 18, 20, 22, 23]. In the equidimensional case, transversality holds at a point $H(p)$ under rather

The first and third authors are supported in part by the NSF grant DMS-0400880. The second author is supported in part by the NSF grant DMS-0401215. 
general conditions. For instance, in [15] it is proved that $H$ is transversal to $M^{\prime}$ at $H(p)$ provided that $M^{\prime}$ is of finite type at $H(p)$ and the generic rank of $\left.H\right|_{\Sigma_{p}}$, where $\Sigma_{p}$ denotes the Segre variety of $M$ at $p$, is $n$. The situation in the case where $n^{\prime}>n$ is much more complicated. Indeed, transversality may fail at a point $H(p)$ even for a polynomial embedding $\mathbb{C}^{2} \rightarrow \mathbb{C}^{3}$ sending one nondegenerate hyperquadric into another, as is illustrated by Example 2.4 below. Observe that a trivial case where transversality fails at all points is when $H(U)$ is contained in $M^{\prime}$. In the equidimensional case, this is the only way for transversality to fail at all points provided that $M$ is holomorphically nondegenerate (see Example 2.2 and Theorem 5.1). When $n^{\prime}>n$, this is no longer the case, as is illustrated by Theorem 1.4 as well as Example 2.5. Our Theorems 1.1 and 1.3 give conditions that guarantee transversality at most points. The results are essentially optimal, as is illustrated by examples. Having tranversality at most points is crucial in the study of rigidity of embeddings into hyperquadrics. See e.g., [4,11-14,16,19,21,25]. See also [1, 26 ] for recent related work on transversality of holomorphic Segre mappings.

Before stating our main results, we introduce some notation. Let $M$ be a hypersurface in $\mathbb{C}^{n+1}, p \in M$, and $\mathcal{L}: \mathbb{C}^{n} \times \mathbb{C}^{n} \rightarrow \mathbb{C}$ a representative of the Levi form of $M$ at $p$. We shall denote by $e(M, p):=\min \left(e_{-}, e_{+}\right)$and $e_{0}(M, p)=e_{0}$, where $e_{+}, e_{-}, e_{0}$, denote the number of positive, negative, and zero eigenvalues of $\mathcal{L}$ at $p$. Observe that $e(M, p)$ and $e_{0}(M, p)$ are independent of the choice of representative $\mathcal{L}$ of the Levi form. A connected hypersurface $M$ is said to be holomorphically nondegenerate if there are no germs of nontrivial holomorphic $(1,0)$-vector fields tangent to $M$. We point out that if $M$ is connected and Levi-nondegenerate at some point, i.e., $e_{0}(M, p)=0$ for some $p \in M$, then $M$ is necessarily holomorphically nondegenerate. The converse is not true. The reader is referred to [3] for further details on this and other related notions (see also [24] for holomorphic nondegeneracy).

In our first theorem, we give two independent conditions guaranteeing transversality at most points.

Theorem 1.1. Let $M \subset \mathbb{C}^{n+1}$, and $M^{\prime} \subset \mathbb{C}^{n^{\prime}+1}$ be connected real-analytic hypersurfaces and $U$ an open neighborhood of $M$ in $\mathbb{C}^{n+1}$. Assume that $M$ is holomorphically nondegenerate and that either

$$
e\left(M^{\prime}, p^{\prime}\right)+e_{0}\left(M^{\prime}, p^{\prime}\right) \leq n-1, \quad \forall p^{\prime} \in M^{\prime}
$$

or

$$
n^{\prime}+e_{0}\left(M^{\prime}, p^{\prime}\right) \leq 2 n, \quad \forall p^{\prime} \in M^{\prime},
$$


Transversality of holomorphic mappings between real hypersurfaces 591

holds. If $H: U \rightarrow \mathbb{C}^{n^{\prime}+1}$ is a holomorphic mapping with $H(M) \subset M^{\prime}$, then one of the following two mutually exclusive conditions holds.

(i) There is an open subset $V \subset U$ with $M \subset V$ and $H(V) \subset M^{\prime}$.

(ii) $H$ is transversal to $M^{\prime}$ at $H(p)$ for all $p \in M$ outside a proper realanalytic subset.

Remark 1.2. We point out that (i) holds if and only if there exists a point $p \in M$ and an open neighborhood $W \subset U$ of $p$ in $\mathbb{C}^{n+1}$ such that $H(W) \subset M^{\prime}$. This follows easily from the connectedness and real-analyticity of $M$. Similarly, (ii) holds if and only if there exists $p \in M$ such that $H$ is transversal to $M^{\prime}$ at $H(p)$. Indeed, if $H$ is transversal at $p \in M$, then we have $\rho^{\prime} \circ H=a \rho$, where $\rho$ and $\rho^{\prime}$ are local real-analytic defining functions near $p$ and $H(p)$, respectively, and $a$ is a real-analytic function defined near $p$, with $a(p) \neq 0$. The set of points, near $p$, at which $H$ is not transversal is given by the equation $a=0$, which defines a proper real-analytic subset of $M$ near $p$. A standard connectedness argument shows that (ii) holds.

The condition (1.2) in Theorem 1.1 is optimal, as can be seen by Example 2.5. Similarly, Theorem 1.4 below shows that condition (1.3) is also optimal. However, if $M$ and $M^{\prime}$ are Levi-nondegenerate and the target is a hyperquadric ${ }^{1}$, then condition (1.3) in Theorem 1.1 can be weakened, as is shown by the following result.

Theorem 1.3. Let $M \subset \mathbb{C}^{n+1}$ be a connected, real-analytic hypersurface and $U$ an open neighborhood of $M$ in $\mathbb{C}^{n+1}$. Let $M^{\prime} \subset \mathbb{C}^{n^{\prime}+1}$ be a nondegenerate hyperquadric. Suppose that $n^{\prime} \leq 3\left(n-e_{0}(M, p)\right)$ for some point $p \in M$. If $H: U \rightarrow \mathbb{C}^{n^{\prime}+1}$ is a holomorphic mapping with $H(M) \subset M^{\prime}$, then one of the following mutually exclusive conditions must hold.

(i) There is an open subset $V \subset U$ with $M \subset V$ and $H(V) \subset M^{\prime}$.

(ii) $H$ is transversal to $M^{\prime}$ at $H(p)$ for all $p \in M$ outside a proper realanalytic subset.

We should remark that conclusion (ii) in Theorems 1.1 and 1.3 cannot be replaced by the stronger conclusion that transversality holds for every $p \in M$, as is shown by Examples 2.3 and 2.4. In the equidimensional case, condition (1.3) is always satisfied. The conclusion of Theorem 1.1, in this

\footnotetext{
${ }^{1}$ By a hyperquadric in $\mathbb{C}^{n+1}$, we mean a real-algebraic hypersurface defined by $\operatorname{Im} w=\langle z, \bar{z}\rangle$, where $\langle\cdot, \cdot\rangle$ is a Hermitian form in $\mathbb{C}^{n}$.
} 
case, can be deduced from known results (e.g., $[7,15]$ ) by using also Theorem 5.1 of the present paper. Even in the equidimensional case, the conclusion (ii) cannot be replaced by that of transversality for all $p \in M$ as is shown by Example 6.2 in [15]. However, if the condition that $M$ is of finite type is added, then it is unknown if this replacement can be made (see [22, Conjecture 2.7]; see also [15, Question 1]).

The following result shows that the condition in Theorem 1.3 requiring $M^{\prime}$ to be a nondegenerate hyperquadric cannot be replaced by the weaker assumption that $M^{\prime}$ is a Levi-nondegenerate hypersurface. As mentioned above, it also shows that the condition (1.3) in Theorem 1.1 is optimal.

Theorem 1.4. Given $M \subset \mathbb{C}^{n+1}$ a nondegenerate hyperquadric, there exist a Levi-nondegenerate hypersurface $M^{\prime} \subset \mathbb{C}^{2 n+2}$ and $H: \mathbb{C}^{n+1} \rightarrow \mathbb{C}^{2 n+2}$ a polynomial embedding of degree 2 such that $H$ sends $M$ into $M^{\prime}$, but neither (i) nor (ii) of Theorem 1.1 holds. More precisely, if $M:=\left\{Z \in \mathbb{C}^{n+1}\right.$ : $\rho(Z, \bar{Z})=0\}$ with

$$
\rho(Z, \bar{Z}):=\operatorname{Im} w-\sum_{j=1}^{n} \delta_{j}\left|z_{j}\right|^{2}, \quad Z=(z, w) \in \mathbb{C}^{n} \times \mathbb{C}
$$

where $\delta_{j}= \pm 1$ and $\langle\cdot, \cdot\rangle$ is a nondegenerate Hermitian form in $\mathbb{C}^{2 n+1}$ with $n$ negative and $(n+1)$ positive eigenvalues, then there exist a polynomial embedding of degree 2, $H: \mathbb{C}^{n+1} \rightarrow \mathbb{C}^{2 n+2}$, and a real bihomogeneous polynomial $\phi\left(z^{\prime}, \bar{z}^{\prime}\right), z^{\prime} \in \mathbb{C}^{2 n+1}$, of bidegree (2,2) such that if

$$
\rho^{\prime}\left(z^{\prime}, w^{\prime}, \bar{z}, \bar{w}\right):=\operatorname{Im} w^{\prime}-\left\langle z^{\prime}, \overline{z^{\prime}}\right\rangle-\phi\left(z^{\prime}, \overline{z^{\prime}}\right)
$$

then $\rho^{\prime} \circ H=-4 \rho^{2}$.

We should point out that if the target hypersurface $M^{\prime}$ in either Theorem 1.1 or 1.3 does not contain any nontrivial complex subvarieties, then condition (i) is equivalent to the mapping $H$ being constant. Hence, if the hypothesis that $M^{\prime}$ does not contain any nontrivial complex subvarieties is added to either Theorem 1.1 or 1.3 and $H$ is assumed to be nonconstant, then the conclusion (ii) necessarily follows. In the last section of this paper, we give a number of sufficient conditions for (i) to hold (see Theorems 5.1 and 5.7 and corollaries). In the equidimensional case (i.e., $n=n^{\prime}$ ), we give two conditions equivalent to (i) (see Corollary 5.2). 
Transversality of holomorphic mappings between real hypersurfaces 593

\section{Examples and a lemma}

In this section, we give some examples, which show that our main results are sharp. We begin with the following lemma, which expresses conditions (i) and (ii) in Theorems 1.1 and 1.3 in terms of local defining functions for $M$ and $M^{\prime}$.

Lemma 2.1. Let $M \subset \mathbb{C}^{n+1}$ and $M^{\prime} \subset \mathbb{C}^{n^{\prime}+1}$ be connected real-analytic hypersurfaces and $U$ an open neighborhood of $M$ in $\mathbb{C}^{n+1}$. Let $p \in M$ and $p^{\prime} \in M^{\prime}$ and suppose that $M$ and $M^{\prime}$ are defined locally by $\rho=0$ and $\rho^{\prime}=0$ near $p$ and $p^{\prime}$, respectively. Let $H: U \rightarrow \mathbb{C}^{n^{\prime}+1}$ be a holomorphic mapping with $H(M) \subset M^{\prime}$ and $H(p)=p^{\prime}$. If (i) in Theorem 1.1 does not hold, then there exists a unique integer $k \geq 1$ such that $\rho^{\prime} \circ H=a \rho^{k}$, where a is a realvalued, real-analytic function defined near $p$ in $\mathbb{C}^{n+1}$ with $\left.a\right|_{M} \not \equiv 0$. Moreover, the condition (ii) in Theorem 1.1 is equivalent to $k=1$.

Proof. Since $H(M) \subset M^{\prime}$ and (i) does not hold, $\rho^{\prime} \circ H$ vanishes on $M$ but is not identically zero near $p$ (by Remark 1.2). Hence, $\rho^{\prime} \circ H=b \rho$, where $b \not \equiv 0$. By unique factorization, there is a unique integer $l \geq 0$ such that $b=a \rho^{l}$ with $\left.a\right|_{M} \not \equiv 0$. Now, (ii) is equivalent to $\left.b\right|_{M} \not \equiv 0$, in view of Remark 1.2 , and hence $k=1+l=1$. The conclusion of the lemma now follows.

Example 2.2. Let $M \subset \mathbb{C}^{2}$ be the Levi-flat hypersurface given by $\rho(z, w$, $\bar{z}, \bar{w}):=\operatorname{Im} w=0$, and $M^{\prime} \subset \mathbb{C}^{2}$ the hypersurface given by $\rho^{\prime}\left(z^{\prime}, w^{\prime}, \bar{z}^{\prime}\right.$, $\left.\bar{w}^{\prime}\right):=\operatorname{Im} w^{\prime}-\left|z^{\prime}\right|^{2}=0$. The mapping $H: \mathbb{C}^{2} \rightarrow \mathbb{C}^{2}$ given by $H(z, w)=$ $\left(w, i w^{2}\right)$ maps $M$ into $M^{\prime}$, but satisfies neither (i) nor (ii) of Theorem 1.1. Indeed, we have $\rho^{\prime} \circ H \equiv-2 \rho^{2}$. Note that $M$ is not holomorphically nondegenerate, which is the only assumption of Theorem 1.1 in this case $\left(n^{\prime}=n=1\right)$.

Example 2.3. Let $M \subset \mathbb{C}^{3}$ be the unit sphere,

$$
\rho(Z, \bar{Z}):=\left|Z_{1}\right|^{2}+\left|Z_{2}\right|^{2}+\left|Z_{3}\right|^{2}-1=0
$$

and $M^{\prime} \subset \mathbb{C}^{5}$ be the hyperquadric defined by

$$
\rho^{\prime}\left(z^{\prime}, w^{\prime}, \bar{z}^{\prime}, \bar{w}^{\prime}\right):=\operatorname{Im} w^{\prime}-\left(\left|z_{1}^{\prime}\right|^{2}+\left|z_{2}^{\prime}\right|^{2}+\left|z_{3}^{\prime}\right|^{2}-\left|z_{4}^{\prime}\right|^{2}\right)=0 .
$$

Consider the mapping

$$
H(Z):=\left(Z_{1} Z_{2}, Z_{2}^{2}, Z_{2} Z_{3}, Z_{2}, 0\right) .
$$


Observe that we have the identity $\rho^{\prime}(H(Z), \overline{H(Z)})=-\left|Z_{2}\right|^{2} \rho(Z, \bar{Z})$. We conclude that $H$ sends $M$ into $M^{\prime}, H\left(\mathbb{C}^{3}\right)$ is not contained in $M^{\prime}$, and $H$ is not transversal to $M^{\prime}$ at 0 . Note also that Theorem 1.1 applies, since condition (1.2) holds. This example shows that, under assumption (1.2), conclusion (ii) in Theorem 1.1 cannot be replaced by the stronger conclusion of transversality at all points of $M$.

Example 2.4. Let $M \subset \mathbb{C}^{2}$ be the hypersurface given by $\rho(z, w, \bar{z}, \bar{w}):=$ $\operatorname{Im} w-|z|^{2}=0, M^{\prime} \subset \mathbb{C}^{3}$ the Levi-nondegenerate hyperquadric given by $\rho^{\prime}\left(z^{\prime}, w^{\prime}, \bar{z}^{\prime}, \bar{w}^{\prime}\right):=\operatorname{Im} w^{\prime}+\left|z_{1}^{\prime}\right|^{2}-\left|z_{2}^{\prime}\right|^{2}=0$ and $H: \mathbb{C}^{2} \rightarrow \mathbb{C}^{3}$ given by

$$
H(z, w)=\left(z+z^{2}+\frac{i}{2} w, z-z^{2}-\frac{i}{2} w,-2 z w\right) .
$$

We have $\rho^{\prime} \circ H \equiv-2(z+\bar{z}) \rho$. Hence $H$ is transversal on $M$ outside the realanalytic submanifold of $M$ given by $\operatorname{Re} z=0$. For every $p^{\prime} \in M^{\prime}$, we have $e_{0}\left(M^{\prime}, p^{\prime}\right)=0$ and $e\left(M^{\prime}, p^{\prime}\right)=1$. Hence, (1.3) in Theorem 1.1 holds (but (1.2) does not). Also, the assumption on $n^{\prime}$ in Theorem 1.3 holds. Moreover, $M$ is holomorphically nondegenerate, (i) does not hold, and transversality does not hold at every point of $M$. This example shows that (ii) in Theorems 1.1 and 1.3 cannot be replaced by the stronger condition of transversality at all points of $M$. Observe that $H\left(\mathbb{C}^{2}\right)$ is the 2-dimensional complex manifold given by $\left(z_{1}^{\prime}+z_{2}^{\prime}\right)\left(z_{1}^{\prime}-z_{2}^{\prime}\right)+i w^{\prime}=\left(z_{1}^{\prime}+z_{2}^{\prime}\right)^{3} / 2$.

Example 2.5. Let $M \subset \mathbb{C}^{2}$ be the hypersurface given by $\rho(z, w, \bar{z}, \bar{w}):=$ $\operatorname{Im} w-|z|^{2}=0, M^{\prime} \subset \mathbb{C}^{5}$ the Levi-nondegenerate hyperquadric given by

$$
\rho^{\prime}\left(z^{\prime}, w^{\prime}, \bar{z}^{\prime}, \bar{w}^{\prime}\right):=\operatorname{Im} w^{\prime}+\left|z_{1}^{\prime}\right|^{2}-\left|z_{2}^{\prime}\right|^{2}-\left|z_{3}^{\prime}\right|^{2}-\left|z_{4}^{\prime}\right|^{2}=0,
$$

and $H: \mathbb{C}^{2} \rightarrow \mathbb{C}^{5}$ given by

$$
H(z, w)=\left(i z+z w,-i z+z w, w, \sqrt{2} z^{2}, i w^{2}\right) .
$$

We have $\rho^{\prime} \circ H \equiv-2 \rho^{2}$. Since neither (i) nor (ii) of Theorem 1.3 holds, this example shows that the condition $n^{\prime} \leq 3\left(n-e_{0}(M, p)\right)$ cannot be replaced by $n^{\prime} \leq 3\left(n-e_{0}(M, p)\right)+1$.

\section{Proof of Theorem 1.1}

For the proof of Theorem 1.1 stated in the introduction, we shall need a number of preliminary results, which may be of independent interest. Recall 
that if $M$ is a real-analytic hypersurface in $\mathbb{C}^{n+1}$, defined locally near $p_{0} \in M$ by the real-analytic equation $\rho(Z, \bar{Z})=0$, then the Segre variety of $M$ at $p$, sufficiently close to $p_{0}$, is given by the holomorphic equation $\rho(Z, \bar{p})=0$. We shall denote the Segre variety of $M$ at $p$ by $\Sigma_{p}$. The following proposition will be useful in the proofs of the main results.

Proposition 3.1. Let $M \subset \mathbb{C}^{n+1}$, and $M^{\prime} \subset \mathbb{C}^{n^{\prime}+1}$ be connected realanalytic hypersurfaces and $U$ an open neighborhood of $M$ in $\mathbb{C}^{n+1}$. If $H$ : $U \rightarrow \mathbb{C}^{n^{\prime}+1}$ is a holomorphic mapping with $H(M) \subset M^{\prime}$ and $M$ is holomorphically nondegenerate, then at least one of the following holds.

(i) There is an open subset $V \subset U$ with $M \subset V$ and $H(V) \subset M^{\prime}$.

(ii) For every $p \in M$ outside a proper real-analytic subset, the rank of $\left.H\right|_{\Sigma_{p}}$ at $p$ is $n$.

Remark 3.2. We note that if, for some point $p \in M$, the restriction of $H$ to the Segre variety of $M$ at $p$ has rank $n$ at $p$, then (ii) in Proposition 3.1 holds. (This is true even without assuming that $M$ is holomorphically nondegenerate.) Indeed, the rank at $p$ of $\left.H\right|_{\Sigma_{p}}$ is the rank of the $n \times\left(n^{\prime}+1\right)$ matrix given by $\left(L_{j} H_{k}(p)\right)$, where $j=1, \ldots, n, k=1, \ldots, n^{\prime}+1$ and $L_{1}, \ldots, L_{n}$ is a real-analytic local basis of the $(1,0)$-vector fields tangent to $M$. Thus, if the rank of this matrix is $n$ at some point, then it is $n$ outside a proper real-analytic subset of $M$ near $p$. A standard connectedness argument shows that (ii) holds.

Proof of Proposition 3.1. We assume, in order to reach a contradiction, that neither (i) nor (ii) of Proposition 3.1 holds. Let $p_{0} \in M$ be a point at which $M$ is finitely nondegenerate ${ }^{2}$, and $\rho, \rho^{\prime}$ local defining functions for $M$ and $M^{\prime}$ near $p_{0}$ and $H\left(p_{0}\right)$, respectively. By Lemma 2.1, there exists an integer $k \geq 1$ such that

$$
\rho^{\prime} \circ H=a \rho^{k}
$$

where $a$ is not identically zero on $M$. By moving to a nearby point, if necessary, we may assume that $a\left(p_{0}\right) \neq 0$. We choose normal coordinates

\footnotetext{
${ }^{2}$ Recall that a real-analytic hypersurface $M \subset \mathbb{C}^{n+1}$ locally defined near a point $p_{0} \in M$ by $\rho(Z, \bar{Z})=0$ is finitely nondegenerate at $p_{0}$ if the vectors $L^{\alpha} \rho_{Z}\left(p_{0}\right), \alpha \in$ $\mathbb{Z}_{+}^{n}$, span all of $\mathbb{C}^{n+1}$, where $\rho_{Z}$ is the gradient vector of $\rho$ with respect to $Z$ and $L^{\alpha}=L_{1}^{\alpha_{1}}, \ldots, L_{n}^{\alpha_{n}}$, with $L_{1}, \ldots, L_{n}$ as in Remark 3.2. If $M$ is connected and holomorphically nondegenerate, then it is finitely nondegenerate on a dense and open subset of $M$ (see $[3,6])$.
} 
$(z, w) \in \mathbb{C}^{n} \times \mathbb{C}$ and $\left(z^{\prime}, w^{\prime}\right) \in \mathbb{C}^{n^{\prime}} \times \mathbb{C}$ for $M$ and $M^{\prime}$ vanishing at $p_{0}$ and $H\left(p_{0}\right)$, respectively. Hence, the defining equations of $M$ and $M^{\prime}$ can be written as $w=Q(z, \bar{z}, \bar{w})$ and $w^{\prime}=Q^{\prime}\left(z^{\prime}, \bar{z}^{\prime}, \bar{w}^{\prime}\right)$, respectively, with $Q(z, 0, \tau) \equiv$ $Q(0, \chi, \tau) \equiv Q^{\prime}\left(z^{\prime}, 0, \tau\right) \equiv Q^{\prime}\left(0, \chi^{\prime}, \tau\right) \equiv \tau$. We write $H(z, w)=(f(z, w)$, $g(z, w))$ with $f=\left(f_{1}, \ldots, f_{n^{\prime}}\right)$. It follows from (3.1) that

$$
g(z, w)-Q^{\prime}(f(z, w), \bar{f}(\chi, \tau), \bar{g}(\chi, \tau))=a(z, w, \chi, \tau)(w-Q(z, \chi, \tau))^{k}
$$

with $a(0) \neq 0$. Setting $\chi=0, \tau=0$, we have $g(z, w)=a(z, w, 0,0) w^{k}$ and hence

$$
g_{w^{k}}(0) \neq 0
$$

We differentiate $(3.2)(k-1)$ times with respect to $w$ and then set $\tau=0$, $w=Q(z, \chi, 0)$. We obtain, since $g(z, 0) \equiv 0$,

$$
\begin{aligned}
g_{w^{k-1}}(z, Q(z, \chi, 0))= & \sum_{\substack{|\alpha| \leq k-1 \\
(3.4)}} Q_{\left(z^{\prime}\right)^{\alpha}}^{\prime}(f(z, Q(z, \chi, 0), \bar{f}(\chi, 0), 0) \\
& \times P_{\alpha}\left(f_{w}\left(z, Q(z, \chi, 0), \ldots, f_{w^{k-1}}(z, Q(z, \chi, 0))\right),\right.
\end{aligned}
$$

where the $P_{\alpha}\left(t_{1}, \ldots, t_{k-1}\right)$ are universal polynomials. We now differentiate (3.4) with respect to $\chi_{j}$, for $1 \leq j \leq n$, and then set $\chi=0$ to obtain

$$
\begin{aligned}
g_{w^{k}}(z, 0) Q_{\chi_{j}}(z, 0,0)= & \sum_{|\alpha| \leq k-1} Q_{\left(z^{\prime}\right)^{\alpha} \chi^{\prime}}^{\prime}(f(z, 0), 0,0) \bar{f}_{\chi_{j}}(0) \\
& \times P_{\alpha}\left(f_{w}(z, 0), \ldots, f_{w^{k-1}}(z, 0)\right) .
\end{aligned}
$$

Since (ii) does not hold, there exist constants $a_{1}, \ldots, a_{n}$ with $\left(a_{1}, \ldots, a_{n}\right) \neq 0$ such that

$$
\sum_{j=1}^{n} \bar{f}_{\chi_{j}}(0) a_{j}=0
$$

Thus, if we multiply (3.5) by $a_{j}$ and sum over $j$, then we obtain

$$
g_{w^{k}}(z, 0) \sum_{j=1}^{n} a_{j} Q_{\chi_{j}}(z, 0,0) \equiv 0 .
$$

It follows from $(3.3)$ that $\sum_{j=1}^{n} a_{j} Q_{\chi_{j}}(z, 0,0) \equiv 0$ and, hence, $\sum_{j=1}^{n} a_{j} Q_{\chi_{j} z^{\alpha}}$ $(0)=0$ for all multi-indices $\alpha$. This contradicts the finite nondegeneracy of $M$ at $p_{0}$ and completes the proof of Proposition 3.1. 
We mention here that some of the techniques in the proof of Proposition 3.1 were used in [4]. The following transversality result may already be known in the folklore. For the reader's convenience, we include a proof here.

Proposition 3.3. Let $M \subset \mathbb{C}^{n+1}$, and $M^{\prime} \subset \mathbb{C}^{n^{\prime}+1}$ be real-analytic hypersurfaces with $p \in M$ and $p^{\prime} \in M^{\prime}$. Let $H:\left(\mathbb{C}^{n+1}, p\right) \rightarrow\left(\mathbb{C}^{n^{\prime}+1}, p^{\prime}\right)$ be a germ at $p$ of a holomorphic mapping sending $M$ into $M^{\prime}$ and such that the restriction of $H$ to the Segre variety of $M$ at $p$ has rank $n$ at $p$. If

$$
e\left(M^{\prime}, p^{\prime}\right)+e_{0}\left(M^{\prime}, p^{\prime}\right) \leq n-1,
$$

then $H$ is transversal to $M^{\prime}$ at $p^{\prime}$.

Proof. We assume, in order to reach a contradiction, that $H$ is not transversal to $M^{\prime}$ at $p^{\prime}$. We choose normal coordinates $(z, w) \in \mathbb{C}^{n} \times \mathbb{C}$ and $\left(z^{\prime}, w^{\prime}\right) \in$ $\mathbb{C}^{n^{\prime}} \times \mathbb{C}$ for $M$ and $M^{\prime}$, vanishing at $p$ and $p^{\prime}$, respectively. We write $H(z, w)=(f(z, w), g(z, w))$ with $f=\left(f_{1}, \ldots, f_{n^{\prime}}\right)$. The defining equations of $M$ and $M^{\prime}$ can be written as $w=Q(z, \bar{z}, \bar{w})$ and $w^{\prime}=Q^{\prime}\left(z^{\prime}, \bar{z}^{\prime}, \bar{w}^{\prime}\right)$, respectively, with $Q(z, 0, \tau) \equiv Q(0, \chi, \tau) \equiv Q^{\prime}\left(z^{\prime}, 0, \tau\right) \equiv Q^{\prime}\left(0, \chi^{\prime}, \tau\right) \equiv \tau$. The fact that $H$ maps $M$ into $M^{\prime}$ implies that

$$
g(z, w)-Q^{\prime}(f(z, w), \bar{f}(\chi, \tau), \bar{g}(\chi, \tau))=a(z, w, \chi, \tau)(w-Q(z, \chi, \tau))
$$

where $a$ is a germ at 0 of a real-analytic function. Since $H$ is not transversal to $M^{\prime}$ at $p^{\prime}$, it follows that $a(0)=0$. Let $v_{j}:=f_{z_{j}}(0)$ for $j=1, \ldots, n$. By assumption, $v_{1}, \ldots, v_{n}$ are linearly independent vectors in $\mathbb{C}^{n^{\prime}}$. We set $w=$ $\tau=0$ in (3.9), apply $\partial^{2} / \partial z_{j} \partial \chi_{l}$ and evaluate at $z=\chi=0$ to obtain

$$
v_{l}^{*} A v_{j}=0, \quad 1 \leq j, l \leq n,
$$

where $A$ is the $n^{\prime} \times n^{\prime}$ Hermitian matrix $\left(Q_{z_{\alpha}^{\prime} \chi_{\beta}^{\prime}}^{\prime}(0)\right)$, the vectors $v_{j}$ are regarded as $n^{\prime} \times 1$ matrices, and ${ }^{*}$ denotes the transpose conjugate. Note that $A$ represents the Levi form of $M^{\prime}$ at $p^{\prime}=H(p)$. We denote the number of positive, negative, and zero eigenvalues of $A$ by $e_{+}, e_{-}, e_{0}$, respectively, and observe that $\min \left(e_{+}, e_{-}\right)=e\left(M^{\prime}, p^{\prime}\right)$ and $e_{0}=e_{0}\left(M^{\prime}, p^{\prime}\right)$. Let $E$ be the $n$-dimensional subspace of $\mathbb{C}^{n^{\prime}}$ spanned by $v_{1}, \ldots, v_{n}$. Let $\mathcal{L}: \mathbb{C}^{n^{\prime}} \times \mathbb{C}^{n^{\prime}} \rightarrow \mathbb{C}$ be the Hermitian form given by $(u, v) \mapsto v^{*} A u$. Equation (3.10) implies that $\mathcal{L}$ restricted to $E \times E$ is identically zero. Standard linear algebra gives $n=\operatorname{dim} E \leq \min \left(e_{+}, e_{-}\right)+e_{0}=e\left(M^{\prime}, p^{\prime}\right)+e_{0}\left(M^{\prime}, p^{\prime}\right)$, contradicting (3.8). This completes the proof of Proposition 3.3. 
For the proof of Theorem 1.1, we shall also need the following proposition.

Proposition 3.4. Let $M \subset \mathbb{C}^{n+1}$, and $M^{\prime} \subset \mathbb{C}^{n^{\prime}+1}$ be connected realanalytic hypersurfaces and $U$ an open neighborhood of $M$ in $\mathbb{C}^{n+1}$. Suppose that

$$
n^{\prime}+e_{0}\left(M^{\prime}, p^{\prime}\right)=2 n, \forall p^{\prime} \in M^{\prime}
$$

holds. Then if $H: U \rightarrow \mathbb{C}^{n^{\prime}+1}$ is a holomorphic mapping with $H(M) \subset M^{\prime}$ such that for every $p \in M$ outside a proper real-analytic subset the restriction of $H$ to the Segre variety of $M$ at $p$ has rank $n$ at $p$, then one of the following mutually exclusive conditions must hold.

(i) There is an open subset $V \subset U$ with $M \subset V$ and $H(V) \subset M^{\prime}$.

(ii) $H$ is transversal to $M^{\prime}$ at $H(p)$ for all $p \in M$ outside a proper realanalytic subset.

Proof. We assume, in order to reach a contradiction, that neither (i) nor (ii) holds. Choose $p_{0} \in M$ such that the restriction of $H$ to $\Sigma_{p_{0}}$ has rank $n$ at $p_{0}$. By Lemma 2.1, there exists an integer $k \geq 2$ such that

$$
\rho^{\prime} \circ H=a \rho^{k}
$$

where $a$ is not identically zero on $M$. By moving to a nearby point, if necessary, we may assume that $a\left(p_{0}\right) \neq 0$. We choose normal coordinates $(z, w) \in$ $\mathbb{C}^{n} \times \mathbb{C}$ and $\left(z^{\prime}, w^{\prime}\right) \in \mathbb{C}^{n^{\prime}} \times \mathbb{C}$ for $M$ and $M^{\prime}$, vanishing at $p_{0}$ and $H\left(p_{0}\right)$, respectively. We write $H(z, w)=(f(z, w), g(z, w))$ with $f=\left(f_{1}, \ldots, f_{n^{\prime}}\right)$. As above, the defining equations of $M$ and $M^{\prime}$ can be written as $w=$ $Q(z, \bar{z}, \bar{w})$ and $w^{\prime}=Q^{\prime}\left(z^{\prime}, \bar{z}^{\prime}, \bar{w}^{\prime}\right)$, respectively. It follows from (3.12) that

$$
g(z, w)-Q^{\prime}(f(z, w), \bar{f}(\chi, \tau), \bar{g}(\chi, \tau))=a(z, w, \chi, \tau)(w-Q(z, \chi, \tau))^{k},
$$

with $a(0) \neq 0$. Let $v_{j}:=f_{z_{j}}(0)$ for $j=1, \ldots, n$. By assumption, $v_{1}, \ldots, v_{n}$ are linearly indepedent vectors in $\mathbb{C}^{n^{\prime}}$. As in the proof of Proposition 3.3, we obtain (3.10), where $A$ is as in that proof. We denote the number of zero eigenvalues of $A$ by $e_{0}$ and observe that $e_{0}=e_{0}\left(M^{\prime}, p_{0}^{\prime}\right)$. We introduce the subspaces $E, F \subset \mathbb{C}^{n^{\prime}}$ spanned by $v_{1}, \ldots, v_{n}$ and $A v_{1}, \ldots, A v_{n}$, 
respectively. Observe that the dimension of $F=A E$ is at least $n-e_{0}$. By equation (3.10), it follows that $E$ and $F$ are orthogonal with respect to the standard Hermitian inner product of $\mathbb{C}^{n^{\prime}}$ and, hence, $E \cap F=\{0\}$. Since $n^{\prime}+e_{0}=2 n$, we conclude that $\mathbb{C}^{n^{\prime}}=E \oplus F$ (and hence the dimension of $F$ is $\left.n-e_{0}\right)$. Let us denote by $v:=f_{w}(0) \in \mathbb{C}^{n^{\prime}}$. By setting $\chi=0, \tau=0$ in (3.13), we conclude that $g(z, w)=a(z, w, 0,0) w^{k}$, and in particular, $g_{w}(0)=$ 0 , since $k \geq 2$. By setting $z=0, \tau=0$ in (3.13), applying $\partial^{2} / \partial w \partial \chi_{j}$, for $j=1, \ldots, n$, and evaluating at 0 , we obtain $v_{j}^{*} A v=\left(A v_{j}\right)^{*} v=0$. Consequently, $v$ is orthogonal to $F$ and, hence, $v \in E$. We set $z=\chi=0$ in (3.13), apply $\partial^{k} / \partial w^{k-1} \partial \tau$, and evaluate at 0 . Since $a(0) \neq 0$, we conclude that

$$
\left.\left(\frac{\partial^{k-1}}{\partial w^{k-1}} Q_{\chi^{\prime}}^{\prime}(f(0, w), 0,0)\right)\right|_{w=0} \bar{v} \neq 0
$$

Similarly, setting $z=0, \tau=0$ in (3.13), applying $\partial^{k} / \partial w^{k-1} \partial \chi_{j}$, for $j=$ $1, \ldots, n$, and evaluating at 0 , we obtain

$$
\left.\left(\frac{\partial^{k-1}}{\partial w^{k-1}} Q_{\chi^{\prime}}^{\prime}(f(0, w), 0,0)\right)\right|_{w=0} \bar{v}_{j}=0, j=1, \ldots, n
$$

Since $v \in E,(3.15)$ contradicts (3.14), completing the proof of Proposition 3.4.

We are now in a position to prove Theorem 1.1.

Proof of Theorem 1.1. In view of Proposition 3.1, we may assume that (ii) of that proposition holds. If condition (1.2) holds, then conclusion (ii) of Theorem 1.1 follows from Proposition 3.3. Thus, to complete the proof, we may assume that condition (1.3) holds. Note that if $n^{\prime}+e_{0}\left(M^{\prime}, p_{0}^{\prime}\right)<2 n$, for some $p_{0}^{\prime} \in M^{\prime}$, then $n^{\prime}+e_{0}\left(M^{\prime}, p^{\prime}\right)<2 n$ holds for all $p^{\prime}$ in an open neighborhood $V$ of $p_{0}^{\prime}$ in $M^{\prime}$ since $p^{\prime} \rightarrow e_{0}\left(M^{\prime}, p^{\prime}\right)$ is lower semicontinuous. Moreover, condition (1.2) then holds for all $p^{\prime} \in V$. Indeed, since $e\left(M^{\prime}, p^{\prime}\right) \leq\left(n^{\prime}-e_{0}\left(M^{\prime}, p^{\prime}\right)\right) / 2$, it follows that $e\left(M^{\prime}, p^{\prime}\right)+e_{0}\left(M^{\prime}, p^{\prime}\right) \leq\left(n^{\prime}+\right.$ $\left.e_{0}\left(M^{\prime}, p^{\prime}\right)\right) / 2<n$ and, hence, (1.2) holds in $V$. The conclusion of Theorem 1.1 follows from Proposition 3.3 (applied to $V$ ) and Remark 1.2. To complete the proof under condition (1.3), we may assume that $n^{\prime}+$ $e_{0}\left(M^{\prime}, p^{\prime}\right)=2 n$ for all $p^{\prime} \in M^{\prime}$. The conclusion of the theorem now follows from Proposition 3.4. 


\section{Proofs of Theorems 1.3 and 1.4}

We now give the proofs of Theorems 1.3 and 1.4.

Proof of Theorem 1.3. We suppose, in order to reach a contradiction, that (i) and (ii) of Theorem 1.3 both fail. Hence, in view of Remark 1.2, we may assume that $H$ is nowhere transversal. Let $p_{0}$ be any point on $M$ at which $e_{0}(M, p)$ is minimal. Note that $e_{0}(M, p)$ is then constant in an open neighborhood of $p_{0}$. Let us, for brevity, denote $e_{0}\left(M, p_{0}\right)$ by $e_{0}$. Let $\rho$ and $\rho^{\prime}$ be local defining functions for $M$ and $M^{\prime}$ near $p_{0}$ and $H\left(p_{0}\right)$, respectively. We conclude, by Lemma 2.1, that there exists an integer $k \geq 2$ and a realanalytic function $a$ defined in a neighborhood of $p_{0}$, not divisible by $\rho$, such that

$$
\rho^{\prime} \circ H=a \rho^{k} .
$$

Since $a \not \equiv 0$ on $M$, we may assume, by moving to a nearby point if necessary, that $a\left(p_{0}\right) \neq 0$. We choose normal coordinates $(z, w) \in \mathbb{C}^{n} \times \mathbb{C}$ and $\left(z^{\prime}, w^{\prime}\right) \in$ $\mathbb{C}^{n^{\prime}} \times \mathbb{C}$ for $M$ and $M^{\prime}$, vanishing at $p_{0}$ and $H\left(p_{0}\right)$, respectively, and write $H(z, w)=(f(z, w), g(z, w))$ with $f=\left(f_{1}, \ldots, f_{n^{\prime}}\right)$. The defining equation of $M^{\prime}$ can be written as

$$
2 i \rho^{\prime}\left(z^{\prime}, w^{\prime}, \bar{z}^{\prime}, \bar{w}^{\prime}\right)=w-\bar{w}-2 i\left\langle z^{\prime}, \bar{z}^{\prime}\right\rangle,
$$

where $\langle\cdot, \cdot\rangle$ is a nondegenerate Hermitian form, and that of $M$ as

$$
2 i \rho(z, w, \bar{z}, \bar{w})=w-\bar{w}-2 i \sum_{j=1}^{n-e_{0}} \delta_{j} z_{j} \bar{z}_{j}+\psi(z, \bar{z}, w+\bar{w}),
$$

where $\psi(z, 0, s)=\psi(0, \bar{z}, s)=0, \psi(z, \bar{z}, s)=\mathrm{O}(3)$, and $\delta_{j}= \pm 1$. Now, identity (4.1) becomes

$$
\begin{aligned}
& g(z, w)-\bar{g}(\chi, \tau)-2 i\langle f(z, w), \bar{f}(\chi, \tau)\rangle \\
& \quad=b(z, w, \chi, \tau)\left(w-\tau-2 i \sum_{j=1}^{n-e_{0}} \delta_{j} z_{j} \chi_{j}+\mathrm{O}(3)\right)^{k},
\end{aligned}
$$

where $b$ is a holomorphic function defined in a neighborhood of 0 in $\mathbb{C}^{2 n+2}$, with $b(0) \neq 0$. We introduce the following vectors in $\mathbb{C}^{n^{\prime}}$

$$
v_{j}:=f_{z_{j}}(0), \quad u_{j}:=f_{z_{j}^{k-2} w}(0), \quad x_{j}:=f_{z_{j} w^{k-1}}(0), \quad j=1, \ldots, n-e_{0},
$$


where the subscripts denote partial derivatives. By carefully identifying appropriate monomials on both sides in (4.4), we conclude that the following identity of $3\left(n-e_{0}\right) \times 3\left(n-e_{0}\right)$ matrices holds:

$$
\left(\begin{array}{ccc}
\left\langle v_{j}, \bar{v}_{l}\right\rangle & \left\langle v_{j}, \bar{u}_{l}\right\rangle & \left\langle v_{j}, \bar{x}_{l}\right\rangle \\
\left\langle u_{j}, \bar{v}_{l}\right\rangle & \left\langle u_{j}, \bar{u}_{l}\right\rangle & \left\langle u_{j}, \bar{x}_{l}\right\rangle \\
\left\langle x_{j}, \bar{v}_{l}\right\rangle & \left\langle x_{j}, \bar{u}_{l}\right\rangle & \left\langle x_{j}, \bar{x}_{l}\right\rangle
\end{array}\right)=\left(\begin{array}{ccc}
0 & 0 & D_{1} \\
0 & D_{2} & A_{1} \\
\bar{D}_{1} & \bar{A}_{1} & A_{2}
\end{array}\right),
$$

where $j, l=1, \ldots, n-e_{0}$ and $D_{1}, D_{2}, A_{1}, A_{2}$ are $\left(n-e_{0}\right) \times\left(n-e_{0}\right)$ matrices. Moreover, $D_{1}$ and $D_{2}$ are invertible diagonal matrices. This proves that the matrix on the left in (4.6) is invertible and, hence, the collection of vectors $v_{1}, \ldots, v_{n-e_{0}}, u_{1}, \ldots, u_{n-e_{0}}, x_{1}, \ldots, x_{n-e_{0}}$, given by $(4.5)$, are linearly independent. Since $n^{\prime} \leq 3\left(n-e_{0}\right)$ by assumption, we conclude that $n^{\prime}=3\left(n-e_{0}\right)$ and the vectors $v_{j}, u_{j}, x_{j}$, for $j=1, \ldots, n-e_{0}$, form a basis in $\mathbb{C}^{n^{\prime}}$. Let now $y:=f_{z_{1}^{k}}(0)$. Again by careful identification of appropriate monomials in (4.4), one can check that $\langle y, \bar{y}\rangle \neq 0$, but

$$
\left\langle y, \bar{v}_{j}\right\rangle=\left\langle y, \bar{u}_{j}\right\rangle=\left\langle y, \bar{x}_{j}\right\rangle=0, j=1, \ldots, n-e_{0} .
$$

This is clearly a contradiction since the vectors $v_{1}, \ldots, v_{n-e_{0}}, u_{1}, \ldots$, $u_{n-e_{0}}, x_{1}, \ldots, x_{n-e_{0}}$ form a basis of $\mathbb{C}^{n^{\prime}}$. This completes the proof of Theorem 1.3.

Proof of Theorem 1.4. We shall take $H(z, w)=(f(z, w), g(z, w))$, with $f=$ $\left(f_{1}, \ldots, f_{2 n+1}\right)$, of the form

$$
f(z, w):=\sum_{j=1}^{n} z_{j} v_{j}+w v_{n+1}+\sum_{j=1}^{n} z_{j} w u_{j}, \quad g(z, w):=2 i w^{2},
$$

where $v_{1}, \ldots, v_{n+1}, u_{1}, \ldots, u_{n}$ are constant linearly independent vectors in $\mathbb{C}^{2 n+1}$ to be determined. We claim that the vectors $v_{1}, \ldots, v_{n+1}, u_{1}, \ldots, u_{n}$ and a bihomogeneous polymomial $\phi\left(z^{\prime}, \bar{z}^{\prime}\right)$ of bidegree $(2,2)$ can be chosen so that

$$
\begin{aligned}
& g(z, w)-\bar{g}(\chi, \tau)-2 i\langle f(z, w), \bar{f}(\chi, \tau)\rangle-2 i \phi(f(z, w), \bar{f}(\chi, \tau)) \\
& =2 i\left(w-\tau-2 i \sum_{j=1}^{n} \delta_{j} z_{j} \chi_{j}\right)^{2}=2 i\left(w^{2}+\tau^{2}-2 w \tau-4 i \sum_{j=1}^{n} \delta_{j} z_{j} \chi_{j} w\right. \\
& \left.\quad+4 i \sum_{j=1}^{n} \delta_{j} z_{j} \chi_{j} \tau-4 \sum_{1 \leq j, k \leq n} \delta_{j} \delta_{k} z_{j} z_{k} \chi_{j} \chi_{k}\right)
\end{aligned}
$$


Let us write $\phi\left(z^{\prime}, \chi^{\prime}\right)$ in the form

$$
\phi\left(z^{\prime}, \chi^{\prime}\right):=T\left(z^{\prime}, \chi^{\prime}, z^{\prime}, \chi^{\prime}\right)
$$

where $T$ is a multilinear form $\mathbb{C}^{2 n+1} \times \mathbb{C}^{2 n+1} \times \mathbb{C}^{2 n+1} \times \mathbb{C}^{2 n+1} \rightarrow \mathbb{C}$ with the symmetries

$$
\begin{gathered}
T\left(X_{1}, Y_{1}, X_{2}, Y_{2}\right)=T\left(X_{2}, Y_{1}, X_{1}, Y_{2}\right)=T\left(X_{1}, Y_{2}, X_{2}, Y_{1}\right) \\
\overline{T\left(X_{1}, Y_{1}, X_{2}, Y_{2}\right)}=T\left(\bar{Y}_{2}, \bar{X}_{2}, \bar{Y}_{1}, \bar{X}_{1}\right)
\end{gathered}
$$

for any $X_{1}, X_{2}, Y_{1}, Y_{2} \in \mathbb{C}^{2 n+1}$.

Our aim is to find vectors $v_{1}, \ldots, v_{n+1}, u_{1}, \ldots, u_{n}$ forming a basis of $\mathbb{C}^{2 n+1}$ and a multilinear form $T$ as above such that (4.8) holds. For this, in view of the choice of $f$ and $g$ given by (4.7), it suffices to establish the two identities:

$$
\begin{gathered}
\langle f(z, w), \bar{f}(\chi, \tau)\rangle=2 w \tau+4 i \sum_{j=1}^{n} \delta_{j} z_{j} \chi_{j} w-4 i \sum_{j=1}^{n} \delta_{j} z_{j} \chi_{j} \tau, \\
T(f(z, w), \bar{f}(\chi, \tau), f(z, w), \bar{f}(\chi, \tau))=4 \sum_{1 \leq j, k \leq n} \delta_{j} \delta_{k} z_{j} z_{k} \chi_{j} \chi_{k} .
\end{gathered}
$$

By carefully identifying all monomials on both sides in (4.11), we conclude that (4.11) holds if and only if the following condition is satisfied:

$$
\begin{aligned}
& \left(\begin{array}{ccc}
\left(\left\langle v_{j}, \bar{v}_{k}\right\rangle\right)_{j, k=1}^{n} & \left(\left\langle v_{j}, \bar{v}_{n+1}\right\rangle\right)_{j=1}^{n} & \left(\left\langle v_{j}, \bar{u}_{k}\right\rangle\right)_{j, k=1}^{n} \\
\left(\left\langle v_{n+1}, \bar{v}_{k}\right\rangle\right)_{k=1}^{n} & \left\langle v_{n+1}, \bar{v}_{n+1}\right\rangle & \left(\left\langle v_{n+1}, \bar{u}_{k}\right\rangle\right)_{k=1}^{n} \\
\left(\left\langle u_{j}, \bar{v}_{k}\right\rangle\right)_{j, k=1}^{n} & \left(\left\langle u_{j}, \bar{v}_{n+1}\right\rangle\right)_{j=1}^{n} & \left(\left\langle u_{j}, \bar{u}_{k}\right\rangle\right)_{j, k=1}^{n}
\end{array}\right) \\
& =\left(\begin{array}{ccc}
0_{n \times n} & 0_{n \times 1} & D \\
0_{1 \times n} & 2 & 0_{1 \times n} \\
\bar{D} & 0_{n \times 1} & 0_{n \times n}
\end{array}\right),
\end{aligned}
$$


Transversality of holomorphic mappings between real hypersurfaces 603 where $D$ is the diagonal $n \times n$ matrix given by

$$
D=\left(\begin{array}{cccc}
-4 i \delta_{1} & 0 & \cdots & 0 \\
0 & -4 i \delta_{2} & \cdots & 0 \\
0 & 0 & \ddots & 0 \\
0 & 0 & \cdots & -4 i \delta_{n}
\end{array}\right)
$$

We must show that there is a basis $v_{1}, \ldots, v_{n+1}, u_{1}, \ldots, u_{n}$ of vectors in $\mathbb{C}^{2 n+1}$ such that (4.13) holds. We note that the eigenvalues of the matrix $\Delta$ on the right in (4.13) are 2 with multiplicity 1,4 with multiplicity $n$, and -4 with multiplicity $n$. Let $e_{1}, \ldots, e_{2 n+1}$ be the standard basis in $\mathbb{C}^{2 n+1}$ and $Q$ the matrix of scalar products

$$
Q=\left(\left\langle e_{\alpha}, \bar{e}_{\beta}\right\rangle\right)_{\alpha, \beta=1}^{2 n+1}
$$

Since $Q$ and $\Delta$ have the same number of positive and negative eigenvalues, there exists an invertible $(2 n+1) \times(2 n+1)$ matrix $A$ such that $\Delta=A Q A^{*}$. If we now let $d_{1}, \ldots, d_{2 n+1}$ be the basis

$$
d_{\alpha}:=\sum_{\gamma=1}^{2 n+1} A_{\alpha \gamma} e_{\gamma}
$$

and set $v_{j}:=d_{j}, j=1, \ldots, n+1$, and $u_{j}:=d_{n+1+j}, j=1, \ldots, n$, then $v_{1}, \ldots, v_{n+1}, u_{1}, \ldots, u_{n}$ is a basis for $\mathbb{C}^{2 n+1}$ that satisfies (4.13).

To determine the multilinear form $T$ to satisfy identity (4.12), we set for $j, k=1, \ldots, n$,

$$
T\left(v_{j}, \bar{v}_{j}, v_{k}, \bar{v}_{k}\right)=T\left(v_{j}, \bar{v}_{k}, v_{k}, \bar{v}_{j}\right)= \begin{cases}2 \delta_{j} \delta_{k}, & \text { if } j \neq k \\ 4, & \text { if } j=k\end{cases}
$$

and $T(X, \bar{Y}, Z, \bar{W})=0$, for all other choices of $X, Y, Z, W$ among the basis vectors $v_{1}, \ldots, v_{n+1}, u_{1}, \ldots, u_{n}$ of $\mathbb{C}^{2 n+1}$. The multilinear mapping $T$ (and hence the bihomogeneous polynomial $\phi$ ) is then uniquely defined by (4.15) and the vanishing condition following that equation. It is then straightforward to check that (4.12) is satisfied. This completes the proof of Theorem 1.4. 


\section{Sufficient conditions for mapping $\mathbb{C}^{n+1}$ into the target hypersurface}

In this section, we give a number of sufficient conditions for conclusion (i) in Theorems 1.1 and 1.3 to hold.

Theorem 5.1. Let $M \subset \mathbb{C}^{n+1}$, and $M^{\prime} \subset \mathbb{C}^{n^{\prime}+1}$ be connected real-analytic hypersurfaces and $U$ an open neighborhood of $M$ in $\mathbb{C}^{n+1}$. Assume that $M$ is holomorphically nondegenerate. If $H: U \rightarrow \mathbb{C}^{n^{\prime}+1}$ is a holomorphic mapping such that $H(M) \subset M^{\prime}$ and the rank of $H$ is $\leq n$ at every $p \in M$, then there is an open subset $V \subset U$ with $M \subset V$ and $H(V) \subset M^{\prime}$.

Proof. We observe that the real rank of $\left.H\right|_{M}$ is $\leq 2 n$, by assumption. We consider first the case where the rank of $\left.H\right|_{M}$ is equal to $2 n$ at some point, and hence on an open and dense subset of $M$. Let $p \in M$ be such a point. We identify $\mathbb{C}^{n+1}$ with $\mathbb{R}^{2 n+2}$ and denote by $h: U \rightarrow \mathbb{R}^{2 n^{\prime}+2}$ the corresponding real-analytic mapping induced by $H$. Note that the rank of $h$ at $p$ is $2 n$, as is the rank of $\left.h\right|_{M}$. It follows that ker $d h(p)$, which is a 2-dimensional subspace of $T_{p} \mathbb{R}^{2 n+2}$, is not contained in the hyperplane $T_{p} M \subset T_{p} \mathbb{R}^{2 n+2}$ and, hence, ker $d h(p)$ is transversal to $T_{p} M$. Consequently, we can find a $2 n$-dimensional submanifold $S \subset M$ through $p$ that is transversal to $\operatorname{ker} d h(p)$ at $p$. By the rank theorem, there is an open neighborhood $W \subset \mathbb{R}^{2 n+2}$ of $p$ such that $h(W)=h(S \cap W)$ and, hence, in particular $h(W) \subset h(M \cap W) \subset M^{\prime}$. The conclusion of the theorem now follows from Remark 1.2.

To complete the proof, we consider now the case where the rank of $\left.H\right|_{M}$ is $\leq 2 n-1$ at every point of $M$. Choose $p_{0} \in M$ such that $M$ is finitely nondegenerate at $p_{0}$ and $\left.H\right|_{M}$ has maximal rank $m \leq 2 n-1$ at $p_{0}$. (This is possible since the points at which $M$ is finitely nondegenerate are dense in $M$.) Let $\omega$ be a small neighborhood of $p_{0}$ in $M$ such that the rank of $\left.H\right|_{M}$ is constant in $\omega$. The image $H(\omega)$ is then a real-analytic submanifold of $\mathbb{C}^{n^{\prime}+1}$. By moving the point $p_{0}$ slightly and shrinking $\omega$ if necessary, we may assume that $H(\omega)$ is a CR submanifold. Since the rank of $\left.H\right|_{M}$ in $\omega$ is $m \leq 2 n-1, H(\omega)$ is CR diffeomeorphic to an $m$-dimensional $\mathrm{CR}$ submanifold of $\omega \subset M$. Since the CR dimension $k$ of $H(\omega)$ is $\leq m / 2$, it follows that $k<n$. In particular, the restriction of $H$ to the Segre variety of $\omega$ at any point in $\omega$ has rank $k<n$. The conclusion of the theorem now follows from Proposition 3.1, as well as Remark 1.2.

The hypothesis that $M$ is holomorphically nondegenerate in Theorem 5.1 cannot be removed, even in the case $n=n^{\prime}$, as is illustrated by Example 2.2. 
We remark that if $M \subset \mathbb{C}^{n+1}$ and $M^{\prime} \subset \mathbb{C}^{n^{\prime}+1}$ are connected realanalytic hypersurfaces, $U$ an open neighborhood of $M$ in $\mathbb{C}^{n+1}$ and $H: U \rightarrow$ $\mathbb{C}^{n^{\prime}+1}$ a holomorphic mapping with $H(M) \subset M^{\prime}$, then (i) in Theorems 1.1 and 1.3 holds if and only if for every $p \in M$ there is an open neighborhod $W$ of $p$ in $\mathbb{C}^{n+1}$ such $H(W) \subset \Sigma_{H(p)}^{\prime}$. Indeed, if $\rho^{\prime}\left(Z^{\prime}, \bar{Z}^{\prime}\right)=0$ is a defining equation for $M^{\prime}$ near $H(p)$, then $H$ sends a full neighborhood of $p$ in $\mathbb{C}^{n+1}$ into $M^{\prime}$ if and only if $\rho^{\prime}(H(Z), \overline{H(Z)}) \equiv 0$. On the other hand, $H$ sends a full neighborhood of $p$ into $\Sigma_{H(p)}^{\prime}$ if and only if $\rho^{\prime}(H(Z), \overline{H(p)}) \equiv 0$. The conclusion above follows easily from these facts. In general, it is not enough to have an open neighborhod $W$ of a single point $p \in M$ such $H(W) \subset \Sigma_{H(p)}^{\prime}$ to conclude that $H$ sends a full neighborhood of $M$ in $\mathbb{C}^{n+1}$ into $M^{\prime}$ as Example 5.4 below illustrates. However, it does suffice in the equidimensional case, as the following straightforward corollary of Theorem 5.1 shows.

Corollary 5.2. Let $M, M^{\prime} \subset \mathbb{C}^{n+1}$ be connected real-analytic hypersurfaces and $U$ an open neighborhood of $M$ in $\mathbb{C}^{n+1}$. Assume that $M$ is holomorphically nondegenerate and let $H: U \rightarrow \mathbb{C}^{n+1}$ be a holomorphic mapping such that $H(M) \subset M^{\prime}$. The following are equivalent:

(i) There is an open subset $V \subset U$ with $M \subset V$ and $H(V) \subset M^{\prime}$.

(ii) There exist $p \in M$ and an open neighborhood $W \subset U$ of $p$ in $\mathbb{C}^{n+1}$ such that $H(M \cap W)$ is contained in the Segre variety of $M^{\prime}$ at $H(p)$.

(iii) The rank of $H$ is $\leq n$ at every $p \in M$.

Another straightforward corollary of Theorem 5.1 is the following.

Corollary 5.3. Let $M \subset \mathbb{C}^{n+1}$ be a real-analytic holomorphically nondegenerate hypersurface with $p \in M, M^{\prime} \subset \mathbb{C}^{N}$ a real-analytic submanifold with $p^{\prime} \in M^{\prime}$, and $H:\left(\mathbb{C}^{n+1}, p\right) \rightarrow\left(\mathbb{C}^{N}, p^{\prime}\right)$ a germ at $p$ of a holomorphic mapping. If there exists a complex subvariety $X \subset \mathbb{C}^{N}$ of dimension $\leq n$ and with $p^{\prime} \in X$, such that $H(M) \subset X \cap M^{\prime}$, then $H\left(\mathbb{C}^{n+1}\right) \subset M^{\prime}$.

Example 5.4. Let $M \subset \mathbb{C}^{2}$ be the unit sphere,

$$
\rho(Z, \bar{Z}):=\left|Z_{1}\right|^{2}+\left|Z_{2}\right|^{2}-1=0,
$$


and $M^{\prime} \subset \mathbb{C}^{3}$ the hypersurface defined by

$$
\rho^{\prime}\left(z^{\prime}, w^{\prime}, \bar{z}^{\prime}, \bar{w}^{\prime}\right):=\operatorname{Im} w^{\prime}-\left|z_{1}^{\prime}\right|^{2}\left(\left|z_{1}^{\prime}\right|^{2}+\left|z_{2}^{\prime}\right|^{2}-1\right)=0
$$

Consider the mapping

$$
H(Z):=\left(Z_{1}, Z_{2}, 0\right)
$$

Observe that we have the identity $\rho^{\prime}(H(Z), \overline{H(Z)})=-\left|Z_{1}\right|^{2} \rho(Z, \bar{Z})$. We conclude that $H$ sends $M$ into $M^{\prime} \cap \Sigma_{H(0,1)}^{\prime}$, where $\Sigma_{H(0,1)}^{\prime}$ denotes the Segre variety of $M^{\prime}$ at $H(0,1)$ and hence (ii) holds. However, neither (i) nor (iii) of Corollary 5.2 holds. This example shows that the conclusion of Corollary 5.2 fails if $M^{\prime}$ is a hypersurface in $\mathbb{C}^{n+2}$ rather than $\mathbb{C}^{n+1}$ as in that corollary.

However, the following result shows that if $M^{\prime}$ is a nondegenerate hyperquadric in $\mathbb{C}^{n+2}$, then the conclusion of Corollary 5.2 still holds.

Corollary 5.5. Let $M \subset \mathbb{C}^{n+1}$ be a connected, real-analytic, holomorphically nondegenerate hypersurface and $U$ an open neighborhood of $M$ in $\mathbb{C}^{n+1}$. Let $M^{\prime} \subset \mathbb{C}^{n+2}$ be a nondegenerate hyperquadric. If $H: U \rightarrow \mathbb{C}^{n+2}$ is a holomorphic mapping such that $H(M)$ is contained in the intersection of $M^{\prime}$ with one of its Segre varieties, then there is an open subset $V \subset U$ with $M \subset V$ and $H(V) \subset M^{\prime}$.

Proof. Let $M^{\prime}$ be given by

$$
\operatorname{Im} w^{\prime}=\sum_{j=1}^{n+1} \delta_{j}\left|z_{j}^{\prime}\right|^{2}, \quad(z, w) \in \mathbb{C}^{n+1} \times \mathbb{C},
$$

where $\delta_{j}= \pm 1$. Without loss of generality, we may assume that $H(M)$ is contained in the intersection of $M^{\prime}$ with the Segre variety of $M^{\prime}$ at 0 , i.e., in the variety given by $w^{\prime}=0$ and $\sum_{j=1}^{n+1} \delta_{j}\left|z_{j}^{\prime}\right|^{2}=0$. Hence, $H=$ $\left(f_{1}, \ldots, f_{n+1}, 0\right)$ where

$$
\sum_{j=1}^{n+1} \delta_{j}\left|f_{j}(Z)\right|^{2}=0, \quad Z \in M
$$

We may assume that $H$ is not constant and hence, after reordering the coordinates if necessary, that $f_{n+1}$ is not identically 0 on $M$. For $Z \in M$ 
Transversality of holomorphic mappings between real hypersurfaces 607 outside the zero set of $f_{n+1}$, we then have

$$
\sum_{j=1}^{n} \delta_{j}\left|\tilde{f}_{j}(Z)\right|^{2}=-\delta_{n+1},
$$

where $\tilde{f}_{j}:=f_{j} / f_{n+1}$. Hence, the mapping $\tilde{H}:=\left(\tilde{f}_{1}, \ldots, \tilde{f}_{n}\right)$ sends $M$, outside the zeros of $f_{n+1}$, into the hyperquadric given by $\sum_{j=1}^{n} \delta_{j}\left|z_{j}^{\prime}\right|^{2}=-\delta_{n+1}$ in $\mathbb{C}^{n}$. By Corollary 5.3, it follows that (5.3) holds identically and, hence, $H$ sends a neighborhood of $M$ in $\mathbb{C}^{n+1}$ into $M^{\prime}$.

Example 5.6. Let $M \subset \mathbb{C}^{2}$ be the unit sphere,

$$
\rho(Z, \bar{Z}):=\left|Z_{1}\right|^{2}+\left|Z_{2}\right|^{2}-1=0,
$$

and $M^{\prime} \subset \mathbb{C}^{4}$ the hyperquadric defined by

$$
\rho^{\prime}\left(z^{\prime}, w^{\prime}, \bar{z}^{\prime}, \bar{w}^{\prime}\right):=\operatorname{Im} w^{\prime}-\left(\left|z_{1}^{\prime}\right|^{2}+\left|z_{2}^{\prime}\right|^{2}-\left|z_{3}^{\prime}\right|^{2}\right)=0 .
$$

Consider the mapping

$$
H(z):=\left(Z_{1} Z_{2}, Z_{2}^{2}, Z_{2}, 0\right)
$$

Observe that we have the identity $\rho^{\prime}(H(Z), \overline{H(Z)})=-\left|Z_{2}\right|^{2} \rho(Z, \bar{Z})$. We conclude that $H$ sends $M$ into $M^{\prime} \cap \Sigma_{0}^{\prime}$, where $\Sigma_{0}^{\prime}=\Sigma_{H(1,0)}^{\prime}$ denotes the Segre variety of $M^{\prime}$ at 0 . Observe that the dimension of $\Sigma_{0}^{\prime}$ is 3 and the CR dimension of $M$ is 1 . Moreover, $H\left(\mathbb{C}^{2}\right)$ is not contained in $M^{\prime}$. This example shows that the conclusion of Corollary 5.5 fails if $M^{\prime}$ is a hyperquadric in $\mathbb{C}^{n+3}$ instead of $\mathbb{C}^{n+2}$.

We conclude this paper by giving another sufficient condition for (i) in Theorem 1.1 to hold. We should point out that a proof of Theorem 5.7 below in the case where $M$ and $M^{\prime}$ are nondegenerate hyperquadrics was given in [4]. We use the notation $e(M, p)$ and $e_{0}(M, p)$ introduced in the introduction.

Theorem 5.7. Let $M \subset \mathbb{C}^{n+1}$, and $M^{\prime} \subset \mathbb{C}^{n^{\prime}+1}$ be connected real-analytic hypersurfaces and $U$ an open neighborhood of $M$ in $\mathbb{C}^{n+1}$. Assume that $M$ 
is holomorphically nondegenerate and

$$
e\left(M^{\prime}, p^{\prime}\right)+e_{0}\left(M^{\prime}, p^{\prime}\right)<\sup _{q \in M} e(M, q), \forall p^{\prime} \in M^{\prime}
$$

If $H: U \rightarrow \mathbb{C}^{n^{\prime}+1}$ is a holomorphic mapping such that $H(M) \subset M^{\prime}$, then there is an open subset $V \subset U$ with $M \subset V$ and $H(V) \subset M^{\prime}$.

Proof. We first observe that (1.2) follows from (5.4), since $e(M, q) \leq n / 2$ for all $q \in M$. It follows from Theorem 1.1 that either (i) or (ii) of that theorem must hold. Thus, to complete the proof of Theorem 5.7, it suffices to show that (ii) cannot hold. Let us assume, in order to reach a contradiction, that (ii) holds. We note that $e(M, p)$ is an integer-valued lower semicontinuous function on $M$. It follows that $e(M, p)=\sup _{q \in M} e(M, q)$ for $p$ in an nonempty open subset of $M$. Hence, we can find a point $p \in M$ such that $H$ is tranversal to $M^{\prime}$ at $p^{\prime}:=H(p)$ and

$$
e\left(M^{\prime}, p^{\prime}\right)+e_{0}\left(M^{\prime}, p^{\prime}\right)<e(M, p)=\sup _{q \in M} e(M, q) .
$$

We choose normal coordinates $(z, w) \in \mathbb{C}^{n} \times \mathbb{C}$ and $\left(z^{\prime}, w^{\prime}\right) \in \mathbb{C}^{n^{\prime}} \times \mathbb{C}$ for $M$ and $M^{\prime}$, vanishing at $p$ and $p^{\prime}$, respectively. We write $H(z, w)=(f(z, w)$, $g(z, w))$ with $f=\left(f_{1}, \ldots, f_{n^{\prime}}\right)$. The defining equations of $M$ and $M^{\prime}$ can be written as $w=Q(z, \bar{z}, \bar{w})$ and $w^{\prime}=Q^{\prime}\left(z^{\prime}, \bar{z}^{\prime}, \bar{w}^{\prime}\right)$, respectively, with $Q(z$, $0, \tau) \equiv Q(0, \chi, \tau) \equiv Q^{\prime}\left(z^{\prime}, 0, \tau\right) \equiv Q^{\prime}\left(0, \chi^{\prime}, \tau\right) \equiv \tau$. The fact that $H$ maps $M$ into $M^{\prime}$ implies that (3.9) in the proof of Proposition 3.3 holds. Since $H$ is transversal to $M^{\prime}$ at $p^{\prime}$, it follows that $a(0) \neq 0$ (see Remark 1.2). Let $v_{j}:=f_{z_{j}}(0)$ for $j=1, \ldots, n$. We let $B$ denote the $n^{\prime} \times n$ matrix whose columns are $v_{1}, \ldots, v_{n}$. We set $w=\tau=0$ in (3.9), apply $\partial^{2} / \partial z_{j} \partial \chi_{l}$ to both sides of (3.9), for $1 \leq j, l \leq n$, and evaluate at $z=\chi=0$ to obtain

$$
B^{*} A^{\prime} B=a(0) A,
$$

where $A^{\prime}$ is the $n^{\prime} \times n^{\prime}$ Hermitian matrix $\left(Q_{z_{i}^{\prime} \chi_{j}^{\prime}}^{\prime}(0)\right), A$ is the $n \times n$ Hermitian matrix $\left(Q_{z_{k} \chi_{l}}(0)\right)$, and ${ }^{*}$ denotes the transpose conjugate. Note that $A$ and $A^{\prime}$ represent the Levi forms of $M$ and $M^{\prime}$ at $p$ and $p^{\prime}$, respectively. Denote by $e_{+}, e_{-}, e_{0}$ and $e_{+}^{\prime}, e_{-}^{\prime}, e_{0}^{\prime}$ the number of positive, negative, and zero eigenvalues of $A$ and $A^{\prime}$, respectively. Recall that $e(M, p)=\min \left(e_{+}, e_{-}\right), e\left(M^{\prime}, p^{\prime}\right)=$ $\min \left(e_{+}^{\prime}, e_{-}^{\prime}\right)$ and $e_{0}\left(M^{\prime}, p^{\prime}\right)=e_{0}^{\prime}$. Thus, the inequality (5.5) implies that $\min \left(e_{+}^{\prime}, e_{-}^{\prime}\right)<\min \left(e_{+}, e_{-}\right)$, which, by standard linear algebra, contradicts identity $(5.6)$ with $a(0) \neq 0$. The proof of Theorem 5.7 is now complete. 
Transversality of holomorphic mappings between real hypersurfaces 609

\section{References}

[1] R. Angle, Some results on holomorphic Segre preserving mappings, Preprint, 2007.

[2] E. Bedford and S. Bell, Proper self-maps of weakly pseudoconvex domains, Math. Ann. 261(1) 1982, 47-49.

[3] M.S. Baouendi, P. Ebenfelt and L.P. Rothschild, Real submanifolds in complex space and their mappings, Princeton Mathematical Series vol. 47. Princeton University Press, Princeton, NJ, 1999 .

[4] M.S. Baouendi and X. Huang, Super-rigidity for holomorphic mappings between hyperquadrics with positive signature, J. Diff. Geom. 69(2) 2005, 379-398.

[5] M.S. Baouendi, X. Huang and L.P. Rothschild, Nonvanishing of the differential of holomorphic mappings at boundary points, Math. Res. Lett. 2(6) 1995, 737-750.

[6] M.S. Baouendi, X. Huang and L.P. Rothschild, Regularity of CR mappings between algebraic hypersurfaces, Invent. Math. 125(1) 1996, $13-36$.

[7] M.S. Baouendi and L.P. Rothschild, Geometric properties of mappings between hypersurfaces in complex space, J. Diff. Geom. 31(2) 1990, 473-499.

[8] M.S. Baouendi and L.P. Rothschild, A generalized complex Hopf lemma and its applications to CR mappings, Invent. Math. 111(2) 1993, $331-348$.

[9] E.M. Chirka and C. Rea, Normal and tangent ranks of CR mappings, Duke Math. J. 76(2) 1994, 417-431.

[10] E.M. Chirka and C. Rea, Differentiable CR mappings and CR orbits, Duke Math. J. 94(2) 1998, 325-340.

[11] J.A. Cima and T.J. Suffridge, A reflection principle with applications to proper holomorphic mappings, Math. Ann. 265(4) 1983, 489-500.

[12] J.P. D'Angelo, Polynomial proper maps between balls, Duke Math. J. 57(1) 1988, 211-219. 
[13] P. Ebenfelt, X. Huang and D. Zaitsev, Rigidity of CRimmersions into spheres, Comm. Anal. Geom. 12(3) 2004, 631-670.

[14] P. Ebenfelt, X. Huang and D. Zaitsev, The equivalence problem and rigidity for hypersurfaces embedded into hyperquadrics, Amer. J. Math. 127 (1) 2005, 169-191.

[15] P. Ebenfelt and L.P. Rothschild, Transversality of CR mappings, Amer. J. Math. 128 2006, 1313-1343.

[16] J.J. Faran, The linearity of proper holomorphic maps between balls in the low codimension case, J. Diff. Geom. 24(1) 1986, 15-17.

[17] J.E. Fornaess, Embedding strictly pseudoconvex domains in convex domains, Amer. J. Math. 98(2) 1976, 529-569.

[18] J.E. Fornaess, Biholomorphic mappings between weakly pseudoconvex domains, Pacific J. Math. 74(1) 1978, 63-65.

[19] F. Forstnerič, Embedding strictly pseudoconvex domains into balls, Trans. Amer. Math. Soc. 295(1) 1986, 347-368.

[20] X. Huang and Y. Pan, Proper holomorphic mappings between real analytic domains in $\mathbf{C}^{n}$, Duke Math. J. 82(2) 1996, 437-446.

[21] X. Huang, On a linearity problem for proper holomorphic maps between balls in complex spaces of different dimensions, J. Diff. Geom. 51(1) 1999, 13-33.

[22] B. Lamel and N. Mir, Remarks on the rank properties of formal cr maps, Sci. China Ser. A 49(11), 2006, 1477-1490.

[23] S.I. Pinčuk, Analytic continuation of mappings along strictly pseudoconvex hypersurfaces, Dokl. Akad. Nauk SSSR, 236(3) 1977, $544-547$.

[24] N.K. Stanton, Infinitesimal CR automorphisms of rigid hypersurfaces, Amer. J. Math. 117(1) 1995, 141-167.

[25] S.M. Webster, The rigidity of $C$ - $R$ hypersurfaces in a sphere, Indiana Univ. Math. J. 28(3) 1979, 405-416.

[26] Y. Zhang, Rigidity and holomorphic Segre transversality for holomorphic Segre maps, Math. Ann. 337(2) 2007, 457-478. 
Transversality of holomorphic mappings between real hypersurfaces 611

Department of Mathematics

University of CALifornia at SAN Diego

LA JOLLA

CA 92093-0112

USA

E-mail address: sbaouendi@ucsd.edu

E-mail address: pebenfel@math.ucsd.edu

E-mail address: lrothschild@ucsd.edu

RECEIVED JANUARY 19, 2007 